\title{
Identification of Molecular Mechanisms Underlying Sex-Associated Differences in the Chronic Obstructive Pulmonary Disease through Bioinformatics Analysis
}

\section{Fengshou Chen}

The First Affiliated Hospital of China Medical University

\section{Haijia Hou}

The First Hospital of China Medical University: The First Affiliated Hospital of China Medical University Jie Han

The First Hospital of China Medical University: The First Affiliated Hospital of China Medical University Bing Tang ( $\nabla$ tangbing527@126.com )

The First Affiliated Hospital of China Medical University

\section{Research}

Keywords: chronic obstructive pulmonary disease, sex differnces, bioinformatics analysis, human, molecular mechanisms

Posted Date: March 26th, 2021

DOI: https://doi.org/10.21203/rs.3.rs-326125/v1

License: (a) (i) This work is licensed under a Creative Commons Attribution 4.0 International License. Read Full License 


\section{Abstract}

Background Accumulating evidence suggests the existence sex associated differences in the Chronic Obstructive Pulmonary Disease (COPD). However, limited knowledge exists on the molecular mechanisms underlying sex associated differences in COPD patients.

Methods The GSE8581 dataset obtained from the GEO database was used to analyze differentially expressed genes (DEGs). Then enrichment analysis for DEGs were conducted through Metascape. PPI and the hub genes-pathway networks were constructed using the STRING database and Cytoscape software. Finally, the CTD was used to examine the relationships between the hub DEGs and COPD.

Results The results revealed that different subsets of DEGs had different characteristics in GO functions and KEGG pathways. Different subsets of hub genes were obtained based on PPI network. The study then constructed the hub genes-pathway network for different subsets to explore the key signaling pathways and hub genes involved. The findings showed that NRAS and RAC1 functioned through "Rap1 signaling pathway" and "PI3K-Akt signaling pathway", in male COPD patients. On the other hand, "Cholesterol metabolism" was among the important pathways in female COPD patients while the hub genes, APOE and APOC3 functioned through "Cholesterol metabolism". Moreover, "Ubiquitin mediated proteolysis" and the "p53 signaling pathway" were shown to play more important roles in male COPD patients compared to their female counterparts. Furthermore, CDK2 and UBE2N were the hub genes involved in "p53 signaling pathway" and "Ubiquitin mediated proteolysis", respectively. Finally the study identified the relationship between the hub genes and COPD in CTD.

Conclusions The present study uncovered different molecular mechanisms in COPD patients based on sex. Additionally, distinct pathways and hub genes including NRAS, RAC1, APOE, APOC3, CDK2 and UBE2N were identified in the two genders of COPD patients. Further studies are needed to explore individualized treatment for COPD based on the findings.

\section{Introduction}

The chronic obstructive pulmonary disease (COPD) is an irreversible or partially reversible disorder with slow progress, characterized by progressive obstruction of airflow [1]. The disease is one of the leading causes of morbidity and mortality across the world [2]. However, the exact molecular and cellular pathogenesis of COPD is yet to be fully elucidated although cigarette smoke (CS) is the predominant risk factor to this complicated process [3]. Notably, numerous studies have been conducted to explore the underlying molecular mechanisms and key genetic factors associated with COPD. For instance, Bhattacharya etal. were the first to report on the changes in the expression of genes in the lung tissue of patients with COPD and provided additional insights into the potential mechanisms [4]. In addition, both chemokines (CCR2, CCL-2, CXCL1) and cytokines (TGF- $\beta$, IFN- $\gamma$, TNF- $\alpha$ ) were reported to be associated with COPD-related changes in the pulmonary system through an LPS COPD model [5]. Moreover, increased serum concentrations of IL-23 and CCL-18 were shown to be correlated with the progression of 
COPD [6]. Nonetheless, there is still no known method for the effective treatment of COPD other than improving the symptoms and delaying disease progression[1].

For a long time, COPD was thought to mostly affect men. However, recent studies uncovered that the rate of COPD was almost simliar in males and females [7, 8]. Nonetheless, extensive evidence highlights sex diferences in the disease. According to a recent study, COPD is expressed differently between women and men with regard to pulmonary function, symptoms, comorbidities and exacerbations [8]. Additionally, a previous study reported that women COPD patients were younger, smoked less, had better $\mathrm{Pa}\left(\mathrm{O}_{2}\right)$, lower $\mathrm{Pa}\left(\mathrm{CO}_{2}\right)$, lower $\mathrm{BMI}$, more exacerbations in the previous year and fewer comorbidities, compared to male COPD patients [9]. Moreover, it was shown that women with lower pack-years of smoking were more likely to report serious dyspnea compared to men [10]. Furthermore, a previous study showed that women COPD patients had a lower mortality rate compared to their male counterparts although both groups had similar causes of death [11]. In addition, management of COPD was reported to differ by sex, with women being more actively managed than men [12].

Accumulating evidence indicates the existence of sex associated diferences in COPD. However, it remains largely unclear how sex may modify these diferences in the condition. In addition, limited knowledge exists on the molecular mechanisms underlying sex differences in COPD patients. Therefore, the present study aimed to uncover the key molecules and related pathways that cause sex-associated differences in COPD patients. These may provide additional insights on the biological characteristics of COPD and shed light on new molecular targets for personalized treatment. An overview of the workflow is shown in Fig. 1.

\section{Materials And Methods}

\section{Microarray Data}

In this study, the GSE8581 dataset was dowloaded from the Gene Expression Omnibus (GEO) database (https://www.ncbi.nlm.nih.gov/geo/) [4]. Notably, 15 COPD lung samples ( 6 females and 9 males) and 19 healthy control (14 females and 5 males) were included in the dataset. In addition, COPD is characteristic by low FEV1/FVC and FEV1 values. Therefore, COPD was identified as subjects with FEV1/FVC $<0.7$ and FEV $1<70 \%$ in the GSE8581 dataset. On the other hand, the controls were considered to be subjects with FEV1/FVC>0.7 and FEV1>80\% $[4,13,14]$.

\section{Analysis of differentially expressed gene}

Background correction was done after which the data was normalized and transformed into a log2-based logarithm using the robust multiarray average algorithm [15-17]. Thereafter, the internal standard probe was removed and a box plot was drawn for the expression values of every chip before and after normalization. In addition, the Linear Models for Microarray Data (limma) package in R was used to analyze 4 subsets of differentially expressed genes (DEGs) [18]. The subsets included COPD vs. NoCOPD, male-COPD vs. male-No-COPD, female-COPD vs. female-No-COPD and male-COPD vs. female COPD. DEGs were considered as genes with a $|\log 2 \mathrm{FC}|>4$ and $p<0.05$. 


\section{Enrichment analysis for the DEGs}

The Gene Ontology (GO) functional and Kyoto Encyclopedia of Genes and Genomes (KEGG) pathway analyses were performed on Metascape which is a powerful annotation tool for the analysis of gene function $[19,20]$. Terms with a $p$ value $<0.05$, an enrichment factor $>1.5$ and a minimum count of 3 were collected and grouped into clusters based on their similarities in membership (Kappa scores $>0.3)[21,22]$. Thereafter, statistically significant terms within a cluster were chosen to represent the cluster. If more than 20 terms for GO or pathway annotations were identified, the top 20 terms were chosen for visualization.

\section{COPD involved genes obtaied from the Phenolyzer}

Phenolyzer (http://phenolyzer.wglab.org/) is a computational tool to implicate genes involved in diseases $[23,24]$. The software workflow can be found at http://phenolyzer.wglab.org/FAQ.php.

\section{Construction of a Protein-protein interaction network}

A protein-protein interaction (PPI) network for overlapping genes between the enriched DEGs from KEGG analysis and COPD related genes from the Phenolyzer was constructed using the STRING database and an interaction score with a median confidence of 0.7 was set as the standard cutoff criterion $[17,25]$. The network was visualized using the Cytoscape software platform (http://www.cytoscape.org/) based on information on functional analysis. Additionally, cytoHubba in Cytoscape, was used to identify the hub genes.

\section{Constructing the hub genes-pathway network}

The Cytoscape software was used to examine the association between hub genes and the hub genesrelated pathways in order to create the hub genes-pathway network. The nodes in different shapes and colors represented different pathways and hub genes in the network.

\section{Identifcation of selected hub DEGs associated with respiratory tract diseases}

The comparative toxicogenomic database (CTD) was used to predict the correlation between diseases and genes [26]. The data abtained from CTD was then used to analyze the association between respiratory tract diseases and gene products.

\section{Results}

\section{Data preprocessing and screening for DEGs}

Following data preprocessing, 4 subsets of DEGs were analyzed, namely; COPD vs. No-COPD, male-COPD vs. male-No-COPD, female-COPD vs. female-No-COPD and male-COPD vs. female-COPD). Thereafter, the study analyzed the global transcriptomic changes in the lung tissues of COPD patients. As a result, a total of 1,442 DEGs, including 808 up-regulated and 634 down-regulated genes, were identified in lung tissues of COPD patients, compared to the healthy controls (Figure 2A-B). The DEGs are listed in 
Supplementary Table S1. Furthermore, 658 up-regulated and 908 down-regulated genes were identified in the lung tissues of male COPD patients, compared to the healthy male controls. On the other hand, 576 up-regulated and 480 down-regulated genes were identified in the lung tissues of female COPD patients, compared to the healthy female controls. Additionally, 1,157 DEGs were identified in the lung tissues of male COPD patients compared to the female ones, including 489 up-regulated and 668 down-regulated genes. The heatmaps and volcano plot showing the gene expression profiles of DEGs are presented in Figure 3C-H. The results presented above therefore show that a considerable number of DEGs in both males and females, is regulated in the lung in response to COPD. The detailed information on DEGs is listed in Supplementary Table S2-4.

The study further investigated whether the genes that were either up-regulated or down-regulated between COPD patients and healthy controls were the same in males and females. Interestingly, only $1.1 \%$ (13 out of 1221) of the genes that were up-regulated and $0.8 \%$ (11 out of 1377) of those that were downregulated in COPD were shared between males and females (Figure 2I-J). The results therefore suggusted that most of the DEGs in male or female COPD patients were different, implicating a diffirent mechanism based on sex, in the disease. The overlapping DEGs are listed in Supplementary Table S5.

\section{Functional annotation for DEGs between all the COPD patients and healthy control samples}

The GO and KEGG analysis of DEGs were performed on Metascape. The results of Go enrichment analysis demonstrated that DEGs between the COPD samples and healthy controls were mainly enriched in "leukocyte migration", "leukocyte activation involved in immune response", "regulation of MAPK cascade", "regulation of T cell activation" and "positive regulation of programmed cell death" (Figure 3A). Addtionally, several pathways, such as the "foxo signaling pathway", "apoptosis", "Retrograde endocannabinoid signaling", "Cytokine-cytokine receptor interaction", the "Chemokine signaling pathway", "NOD-like receptor signaling pathway" and "p53 signaling pathway", were enriched in the DEGs of COPD patients (Figure 3B). The results are demonstrated in Supplementary Table S6-7.

\section{Functional annotation of the different subsets of DEGs in male and female COPD patients}

The results revealed a clear difference in the GO and KEGG pathway terms between the male and female COPD patients (Figure 4). Notably, the DEGs in male patients were enriched in the GO terms related to "positive regulation of apoptotic process", "negative regulation of phosphorylation" and "positive regulation of NK T cell activation". On the other hand, the DEGs in female COPD patients were mainly enriched in "negative regulation of immune effector process" and "regulation of immune effector process" (Figure 4A-B). Interestingly, the DEGs in male COPD patients were enriched in such pathways as the "RIG-I-like receptor signaling pathway", "GnRH signaling pathway", "p53 signaling pathway" and "PI3K-Akt signaling pathway". However, the DEGs in female COPD patients were enriched in pathways including "cholesterol metabolism", "Glutamatergic synapse" and "Hippo signaling pathway" (Figure 4DE). Additionally, the DEGs in both male and female COPD sampls were enriched in several GO terms, such as the "cellular lipid catabolic process", "lipid biosynthetic process", "hormone biosynthetic process" and "regulation of hormone levels" (Figure 4C). Moreover, the DEGs in both male and female COPD samples 
were mainly enriched in pathways such as the "PPAR signaling pathway", "AMPK signaling pathway", "Calcium signaling pathway" and "p53 signaling pathway" (Figure 4F). The results are demonstrated in Supplementary Table S8-13.

\section{Construction of a PPI network for the enriched DEGs from KEGG analysis and selection of hub genes}

We detected 14,211 genes associated with COPD from the Phenolyzer, and 121 genes of those genes belonged to seed genes(Supplementary Table S14). 336, 137 and 193 overlapping DEGs for male COPD patients compared to healthy male controls, female COPD patients compared to healthy female controls and male COPD patients compared to female COPD patients, were obtained between these 14,211 genes associated with COPD from the Phenolyzer and the enriched DEGs from KEGG analysis(Supplementary Table S15).

A PPI network of the different subsets of above overlapping DEGs was constructed in order to distinguish hub genes from the common one(Figure 5-7). The top ten degree genes in the PPI network were identified as the hub genes. The following were identified as the hub genes in the lung tissues of male COPD patients compared to healthy male controls; NRAS, RAC1, ATG7, UBE2N, SNRPF, DHX38, SNRPD1, HNRNPC, SRSF5 and CPSF1. On the other hand, the following were identified as the hub genes in the lung tissues of female COPD patients compared to healthy female controls; APOE, GNGT2, FGA, FGG, ADCY2, C5, HEXB, PDYN, APOC3 and PLA2G7. Notably, the analysis revealed that male and female COPD patients had different key genes. In addition, UBE2N, CDK2, ANAPC2, CDC16, ANAPC4, ANAPC7, UBE2R2, HUWE1, UBE4A and KLHL13 were identified as the unique hub genes between the male and female COPD patients.

\section{Construction of the hub genes-pathway network}

The hub genes-pathway network for the different subsets was constructed to further explore the key signaling pathways in which the selected hub genes were involved (Figure 8 ). Therefore, using the degree method in cytoHubba, the 10 genes with the highest degrees were identified as hub genes in the network. In the PPI network, it was shown that one hub gene was associated with different pathways while one pathway connected to several hub genes. In addition, the results showed that NRAS and RAC1 functioned through the "Rap1 signaling pathway" and "PI3K-Akt signaling pathway", in male COPD patients. This suggested that NRAS, RAC1, the "Rap1 signaling pathway" and "PI3K-Akt signaling pathway" played an important in the mechanisms of COPD in male patients (Figure 8A and Supplementary Figure 1). On the other hand, "Cholesterol metabolism" was one of the important pathways in female COPD patients and the hub genes, APOE and APOC3, functioned through "Cholesterol metabolism" (Figure 8B and Supplementary Figure 2). Moreover, "Ubiquitin mediated proteolysis" and the "p53 signaling pathway" played more important roles in male COPD patients compared to the females. Furthermore, CDK2 and UBE2N were the hub genes involved in the "p53 signaling pathway" and "Ubiquitin mediated proteolysis", respectively (Figure $8 \mathrm{C}$ and Supplementary Figure 3 ). 
In addition, the CTD database showed that NRAS, RAC1, APOE, APOC3, UBE2N and CDK2 involved several respiratory tract diseases including COPD (NRAS: inference score 26.9; RAC1: inference score 36.5; APOE: inference score 30.57; APOC3: inference score 13.71; UBE2N: inference score 19.94; CDK2: inference score 15.99). This data is shown in Figure 9.

\section{Discussion}

The present study sought to explore the sex associated differences in the expression of genes in the human lung tissues using whole-genome microarrays, to clarify the underlying molecular mechanisms. Therefore, the study first conducted bioinformatics analysis of DEGs in male and female COPD patients (COPD vs. No-COPD, male-COPD vs. male-No-COPD, female-COPD vs. female-No-COPD, male-COPD vs. female -COPD). The results showed that the 4 subsets of DEGs had different characteristics in GO function and KEGG pathways. In addition, Go enrichment analysis demonstrated that the DEGs between the healthy controls and COPD samples were mainly enriched in "leukocyte migration", "leukocyte activation involved in immune response", "regulation of T cell activation" and "positive regulation of programmed cell death". In addition, the "Foxo signaling pathway", "apoptosis", "Cytokine-cytokine receptor interaction", "Chemokine signaling pathway" and "NOD-like receptor signaling pathway" were enriched in the DEGs of COPD patients. Moreover, there was a clear difference in the GO functional and KEGG pathway terms between the male and female COPD patients. Notably, the following GO terms were enriched in male COPD patients; "positive regulation of apoptotic process", "negative regulation of phosphorylation" and "positive regulation of NK T cell activation". However, the DEGs in female COPD patients were mainly involved in "negative regulation of immune effector process" and "regulation of immune effector process". Moreover, the DEGs in male COPD patients were enriched in pathways suh as the "p53 signaling pathway" and "PI3K-Akt signaling pathway", while those in female COPD patients were involved in such pathways as "Cholesterol metabolism" and "Glutamatergic synapse". Additionally, the DEGs in male and female COPD samples were enriched in several GO terms, including "regulation of hormone levels", "hormone biosynthetic process" and "lipid biosynthetic process". The DEGs in the male and the female COPD samples were also mainly enriched in pathways such as the "Cell cycle", "p53 signaling pathway" and "Ubiquitin mediated proteolysis". Furthermore, the hub genes in the different subsets were obtained based on the PPI network for the enriched DEGs from KEGG analysis. Finally, the study constructed the hub genes-pathway network for the different subsets to explore the key signaling pathways and the hub genes involved.

In the present study, NRAS and RAC1 were identified as the hub genes in the PPI network of DEGs in male COPD patients. NRAS is one of the 3 members of the RAS oncogene family and encodes small GTP enzymes that play a role in cellular signal transduction [27]. In addition, it was reported to potentially play a role in the regulation of angiogenesis, cancer cell survival, invasion, migration and growth in various types of cancer $[27,28]$. Moreover, a previous study reported that the levels of NRAS proteins in the lung homogenates of rats ( 20 days or older) increased 5 to 6 -fold, compared to fetal levels. Additionally, localization of the NRAS protein was restricted to keratin expressing cells in bronchiolar structures, partcularly in ciliated cells [29]. On the other hand, RAC1 is a member of the Rho family of small GTP 
enzymes which are known to act as molecular switches that regulate numerous important cellular processes. Furthermore, RAC1 is an expressed signal transducer that regulates several processes related to inflammatory reactions, including cell adhesion, chemotaxis as well as vascular permeability [30] and has been extensively studied in lung diseases. According to a previous report, pharmacological inhibition or deletion of RAC1 in airway smooth muscle cells prevented airway hyperresponsiveness in murine models of allergic asthma [31]. In addition, impaired branching and decreased expression of AXIN2 and FGFR2 $b$ was induced through the inhibition of RAC1 to regulate the morphogenesis of lung branching [32]. Moreover, the selective inhibition of RAC1 not only ameliorated the release of IL-1 $\beta$ and inflammation in model lungs but also reduced the infiltration of neutrophils and macrophages into the airway lumen [33]. In addition, RAC1 was shown to be involved in CS-mediated epithelial-mesenchymal transition and lipopolysaccharide-induced pulmonary injury [34]. A previous study also showed that knockdown or inhibition of RAC1 decreased the release of IL- 8 and IL-6 in 16HBE cells induced by the CS extract, which was associated with the CS extract-induced RAC1-regulated signaling pathway [30]. Additionally, NRAS and RAC1 are involved in the "PI3K-Akt signaling pathway" [35,36], which was shown to play a significant role in COPD [37]. According to a previous report, the mutant NRAS protein constitutively activates downstream signaling cascades such as PI3K/AKT/mTOR, Ral pathways and MAPK, resulting in uncontrolled cell proliferation and tumor growth [35]. Furthermore, thrombin was reported to activate the RAC1/PI3K/Akt pathway in human lung epithelial cells, which in turn induces the expression of IL8/CXCL8, transactivation of NF-KB and activation IKKa/ $\beta$ [36]. In addition, activation of PI3K was shown to reduce the apoptosis of alveolar epithelial cells in an in vitro COPD model by inducing the $\mathrm{PI3K} / \mathrm{AKT} / \mathrm{mTOR}$ pathway [37]. Additionally, Chen et al. showed that miRNA1013p.1 enhanced the progression of COPD by activating the EGFR/PI3K/AKT signaling pathway [38]. Notably, the "Rap1 signaling pathway" has been studied in lung diseases and NRAS along with RAC1 were proven to be related with Rap1. Moreover, knockdown of the Rap1 signaling pathway inhibited the protective effect of iloprost against pulmonary endothelial barrier dysfunction and ventilator-induced lung injury [39]. It is noteworthy that NRAS and Rap1 both belong to the Ras family [40]. Additionally, RAC1 activity is required for adenosine-induced enhancement of the endothelial cell barrier. However, activation of RAC1 and the ability to strengthen the endothelial cell barrier depends on the cAMP-dependent Rap1 signaling pathway [41]. Birukova et al. also showed that knockdown of Rap1 through the siRNA approach decreased prostaglandin-induced enhancement of the endothelial cell barrier and RAC1 activation [42]. The present study also found that NRAS and RAC1 functioned through the Rap1 and PI3K-Akt signaling pathways in male COPD patients. Therefore, combined with the findings from previous studies, the results herein implicated the involvement and importance of these two hub genes and two pathways in male COPD patients.

Moreover, the study identified "Cholesterol metabolism" as one of the main pathways in female COPD patients. A previous study reported that changes in the level of proteins in cholesterol metabolism were involved in acute exacerbation of COPD in patients [43]. Additionally, it was shown that mice deficient in $\mathrm{CH} 25 \mathrm{H}$, an enzyme crucial for the metabolism of cholesterol, failed to generate inducible bronchusassociated lymphoid tissue in their lungs, following exposure to chronic CS and were protected against 
the progression of COPD [44]. As a type of an apolipoprotein, APOE mediates the transport of triglycerideand cholesterol-rich lipoprotein particles into cells through receptor-mediated endocytosis. It is also expressed by lung cells to modulate normal lung health and the pathogenesis of respiratory diseases through APOE /LDLR-dependent pathways [45]. In APOE (-/-) mice, there was an increase in bronchoalveolar lavage fluid analytes involved in functions such as tissue remodeling, cell proliferation and inflammation [46]. Additionally, abnormal cholesterol efflux increased systemic inflammation with subsequent emphysema and lung damage in APOE (-/-) mice subjected to an atherogenic diet [47]. On the other hand, APOC3 is an apolipoprotein in the blood, with a pivotal role in triglyceride metabolism [48]. A previous study reported elevated levels of non-esterified fatty acids, cholesterols and triglycerides in the plasma of APOC3-transgenic mice [49]. Furthermore, Volanesorsen was shown to reduce APOC3 which in turn reduced total cholesterol, TG levels and accumulation of hepatic lipids [50]. Although APOE and APOC3 are involved in "Cholesterol metabolism", both hub genes are yet to be studied in COPD. In this study, both APOE and APOC3 were shown to function through "Cholesterol metabolism" in the hub genespathway network of female COPD patients, highlighting the significant role of APOE and APOC3 in female COPD patients.

Loss of diaphragm muscle strength in inflammatory lung diseases including COPD is usually associated with diaphragm fiber atrophy and contributes to mortality [51]. Patients with COPD also develop skeletal muscle atrophy [52]. In addition, a previous study showed that Ubiquitin (Ub) 26S-proteasome System (UPS)-dependent protein breakdown mediates muscle atrophy and was elevated in the diaphragm muscle of patients with COPD [51]. Moreover, activation of the ubiquitin-proteasome pathway was related to atrophy in limb muscles and enhanced protein degradation in COPD patients [53]. In the present study, the results demonstrated that "Ubiquitin mediated proteolysis" played a more important role in male COPD patients. Additionally, UBE2N was selected as a hub gene in ubiquitin mediated proteolysis pathway, based on the hub genes-pathway network. Notably, UBE2N plays a significant role in the regulation of inflammatory and immune pathways [54]. Nonetheless, the role of UBE2N in ubiquitin mediated proteolysis and COPD is yet to studied and therefore needs to be explored further. The present study also found that the "p53 signaling pathway" played a more important role in male COPD patients compared to the females. P53 triggers anti-inflammatory responses in the lungs, suggesting its potential therapeutic role in pulmonary inflammatory diseases [55]. Furthermore, polymorphisms in the P53 gene were associated with apoptotic signaling and CS-related emphysematous changes in the lungs of smokers [56]. Additonal studies also showed that miR-150 suppresses CS-induced apoptosis of airway epithelial cells and lung inflammation, partly through the repression of p53 [57]. Li et al. also showed that LncRNA MEG3 mediates PM2.5-induced cell apoptosis and autophagy, probably through regulating the expression of p53 [58]. In addition, senescence of Type II alveolar epithelial cells was shown to be regulated through the LncRNAmediated FoxO3a and SIRT1/p53 signaling pathways in the pathogenesis of COPD [59]. Moreover, this study identified CDK2 as one of the hub genes involved in the "p53 signaling pathway", from PPI analysis and the hub genes-pathway network. As a member of the Ser/Thr protein kinase family, CDK2 phosphorylates p53 and activates the downstream signaling pathways [60]. The results from this study also suggested that CDK2 exerted more effects in male COPD patients compared 
to their female counterparts. More studies are however needed to explore the effects of CDK2 and the "p53 signaling pathway", especially in male COPD patients.

While the study uncovered some insightful findings, it had a number of limitations. First,this was a microarray analysis and all the results were based on gene expression values. In addition, the sample size was relatively small and a large-sample, multicentre study is therefore needed in future. Second, although the DEGs screened in this study were associated with sex differences in COPD, further validation through in vivo and in vitro experiments as well as through clinical cases, is needed to confirm the results. Additionally, it is necessary to perform functional studies to confirm the roles of the DEGs in the sex associated differences in COPD.

\section{Conclusion}

The present study indicated that different molecular mechanisms in COPD patients based on sex. Additionally, distinct pathways and hub genes including NRAS, RAC1, APOE, APOC3, CDK2 and UBE2N were identified in the two genders of COPD patients. The findings from the present study therefore provide insights on the mechanisms underlying sex associated differences in COPD and may suggest potential therapeutic targets for disease treatment. Further studies are needed to explore individualized treatment for COPD based on these sex associated different pathways and hub genes in future.

\section{Declarations}

\section{Ethics approval and consent to participate}

Not applicable.

\section{Consent for publication}

Not applicable.

\section{Availability of supporting data}

The GSE8581 dataset was downloaded from GEO (http://www.ncbi.nlm.nih.gov/geo/) [4]. In addition, the expression profiling arrays were generated using GPL570[HG-U133_Plus_2] Affymetrix Human Genome U133 Plus 2.0 Array. Other datasets supporting the conclusions in this article are included within the article and its additional files.

\section{Competing interests}

The authors report no conflicts of interest and are solely responsible for the content and writing of this manuscript.

\section{Funding}


None

\section{Authors' contributions}

FSC, HJH and BT were responsible for all aspects regarding the reliability and freedom from bias of the data presented. They were also charged with interpretation of the data and drafting the manuscript. In addition, FSC, BT, DW, HJH and JH were in charge of statistical analyses and interpretation of the data. Finally, BT, HJH and $\mathrm{JH}$ were responsible for evaluation of the full text and guidance as well as final approval of the version to be submitted. Nonetheless, all the authors read and approved the fnal manuscript.

\section{Acknowledgements}

We would like to acknowledge the reviewers for their invaluable comments on this study.

\section{References}

1. Zhao J, Cheng W, He X, Liu Y, Li J, Sun J, et al. Chronic Obstructive Pulmonary Disease Molecular Subtyping and Pathway Deviation-Based Candidate Gene Identification. Cell journal. 2018;20:32632.

2. Zhu M, Ye M, Wang J, Ye L, Jin M. Construction of Potential miRNA-mRNA Regulatory Network in COPD Plasma by Bioinformatics Analysis. Int J Chronic Obstr Pulm Dis. 2020;15:2135-45.

3. Lin $Y Z$, Zhong XN, Chen X, Liang Y, Zhang H, Zhu DL. Roundabout signaling pathway involved in the pathogenesis of COPD by integrative bioinformatics analysis. Int $\mathrm{J}$ Chronic Obstr Pulm Dis. 2019;14:2145-62.

4. Bhattacharya S, Srisuma S, Demeo DL, Shapiro SD, Bueno R, Silverman EK, et al. Molecular biomarkers for quantitative and discrete COPD phenotypes. Am J Respir Cell Mol Biol. 2009;40:35967.

5. Lee SY, Cho SS, Bae CS, Bae MS, Park DH. Socheongryongtang suppresses COPD-related changes in the pulmonary system through both cytokines and chemokines in a LPS COPD model. Pharmaceutical biology. 2020;58:538-44.

6. Rong B, Fu T, Rong C, Liu W, Li K, Liu H. Association between serum CCL-18 and IL-23 concentrations and disease progression of chronic obstructive pulmonary disease. Scientific reports. 2020;10:17756.

7. Choi JY, Kim SY, Lee JH, Park YB, Kim YH, Um SJ, et al. Clinical Characteristics of Chronic Obstructive Pulmonary Disease in Female Patients: Findings from a KOCOSS Cohort. Int $\mathrm{J}$ Chronic Obstr Pulm Dis. 2020;15:2217-24.

8. Perez TA, Castillo EG, Ancochea J, Pastor Sanz MT, Almagro P, Martínez-Camblor P, et al. Sex differences between women and men with COPD: A new analysis of the 3CIA study. Respiratory medicine. 2020;171:106105. 
9. Jia G, Lu M, Wu R, Chen Y, Yao W. Gender difference on the knowledge, attitude, and practice of COPD diagnosis and treatment: a national, multicenter, cross-sectional survey in China. Int J Chronic Obstr Pulm Dis. 2018;13:3269-80.

10. Watson L, Vestbo J, Postma DS, Decramer M, Rennard S, Kiri VA, et al. Gender differences in the management and experience of Chronic Obstructive Pulmonary Disease. Respiratory medicine. 2004;98:1207-13.

11. Celli B, Vestbo J, Jenkins CR, Jones PW, Ferguson GT, Calverley PM, et al. Sex differences in mortality and clinical expressions of patients with chronic obstructive pulmonary disease. The TORCH experience. Am J Respir Crit Care Med. 2011;183:317-22.

12. Åberg J, Hasselgren M, Montgomery S, Lisspers K, Ställberg B, Janson C, et al. Sex-related differences in management of Swedish patients with a clinical diagnosis of chronic obstructive pulmonary disease. Int J Chronic Obstr Pulm Dis. 2019;14:961-9.

13. Heo IR, Kim HC, Kim TH. Health-Related Quality of Life and Related Factors in Persons with Preserved Ratio Impaired Spirometry: Data from the Korea National Health and Nutrition Examination Surve. Medicina (Kaunas, Lithuania). 2020; 57.

14. Çolak Y, Afzal S, Nordestgaard BG, Lange P, Vestbo J. Importance of Early COPD In Young Adults for Development of Clinical COPD: Findings from the Copenhagen General Population Study. American journal of respiratory and critical care medicine. 2020.

15. Chen H, Chen C, Yuan X, Xu W, Yang MQ, Li Q, et al. Identification of Immune Cell Landscape and Construction of a Novel Diagnostic Nomogram for Crohn's Disease. Frontiers in genetics. 2020;11:423.

16. Xu WH, Wu J, Wang J, Wan FN, Wang HK, Cao DL, et al. Screening and Identification of Potential Prognostic Biomarkers in Adrenocortical Carcinoma. Frontiers in genetics. 2019;10:821.

17. Ma L, Lu H, Chen R, Wu M, Jin Y, Zhang J, et al. Identification of Key Genes and Potential New Biomarkers for Ovarian Aging: A Study Based on RNA-Sequencing Data. Frontiers in genetics. 2020;11:590660.

18. Ritchie ME, Phipson B, Wu D, Hu Y, Law CW, Shi W, et al. limma powers differential expression analyses for RNA-sequencing and microarray studies. Nucleic acids research. 2015;43:e47.

19. Zhang YF, Meng LB, Hao ML, Yang JF, Zou T. Identification of Co-expressed Genes Between Atrial Fibrillation and Stroke. Front Neurol. 2020;11:184.

20. Liang B, Li C, Zhao J. Identification of key pathways and genes in colorectal cancer using bioinformatics analysis. 33. London: Medical oncology (Northwood; 2016. p. 111.

21. Niu X, Zhang J, Zhang L, Hou Y, Pu S, Chu A, et al. Weighted Gene Co-Expression Network Analysis Identifies Critical Genes in the Development of Heart Failure After Acute Myocardial Infarction. Frontiers in genetics. 2019;10:1214.

22. Qi L, Liu B, Chen X, Liu Q, Li W, Lv B, et al. Single-Cell Transcriptomic Analysis Reveals Mitochondrial Dynamics in Oocytes of Patients With Polycystic Ovary Syndrome. Frontiers in genetics. 2020;11:396. 
23. Yang H, Robinson PN, Wang K. Phenolyzer: phenotype-based prioritization of candidate genes for human diseases. Nature methods. 2015;12:841-3.

24. Odgerel Z, Sonti S, Hernandez N, Park J, Ottman R, Louis ED, et al. Whole genome sequencing and rare variant analysis in essential tremor families. PloS one. 2019;14:e0220512.

25. Kumari N, Karmakar A, Chakrabarti S, Ganesan SK. Integrative Computational Approach Revealed Crucial Genes Associated With Different Stages of Diabetic Retinopathy. Frontiers in genetics. 2020;11:576442.

26. Qiu Y, Hao ML, Cheng XT, Hua Z. Bioinformatics Analysis of Genes and Mechanisms in Postherpetic Neuralgia. Pain research \& management. 2020; 2020: 1380504.

27. Liu X, Lv X, Yang Q, Jin H, Zhou W, Fan Q. MicroRNA-29a Functions as a Tumor Suppressor and Increases Cisplatin Sensitivity by Targeting NRAS in Lung Cancer. Technology in cancer research treatment. 2018;17:1533033818758905.

28. Cantley LC. The phosphoinositide 3-kinase pathway. 296. New York: Science; 2002. pp. 1655-7.

29. Thrane EV, Becher R, Låg M, Refsnes M, Huitfeldt HS, Schwarze PE. Differential distribution and increased levels of ras proteins during lung development. Experimental lung research. 1997;23:3549.

30. Jiang JX, Zhang SJ, Shen HJ, Guan Y, Liu Q, Zhao W, et al. Rac1 signaling regulates cigarette smokeinduced inflammation in the lung via the Erk1/2 MAPK and STAT3 pathways. Biochimica et biophysica acta Molecular basis of disease. 2017; 1863: 1778-88.

31. André-Grégoire G, Dilasser F, Chesné J, Braza F, Magnan A, Loirand G, et al. Targeting of Rac1 prevents bronchoconstriction and airway hyperresponsiveness. The Journal of allergy and clinical immunology. 2018; 142: 824 - 33.e3.

32. Danopoulos S, Krainock M, Toubat O, Thornton M, Grubbs B, Al Alam D. Rac1 modulates mammalian lung branching morphogenesis in part through canonical Wnt signaling. American journal of physiology Lung cellular molecular physiology. 2016;311:L1036-149.

33. Zhang S, Zhang W, Zeng X, Zhao W, Wang Z, Dong X, et al. Inhibition of Rac1 activity alleviates PM2.5-induced pulmonary inflammation via the AKT signaling pathway. Toxicology letters. 2019;310:61-9.

34. Shen HJ, Sun YH, Zhang SJ, Jiang JX, Dong XW, Jia YL, et al. Cigarette smoke-induced alveolar epithelial-mesenchymal transition is mediated by Rac1 activation. Biochim Biophys Acta. 2014;1840:1838-49.

35. Vujic I, Sanlorenzo M, Posch C, Esteve-Puig R, Yen AJ, Kwong A, et al. Metformin and trametinib have synergistic effects on cell viability and tumor growth in NRAS mutant cancer. Oncotarget. 2015;6:969-78.

36. Lin $\mathrm{CH}$, Cheng HW, Ma HP, Wu CH, Hong $\mathrm{CY}$, Chen BC. Thrombin induces NF-kappaB activation and IL-8/CXCL8 expression in lung epithelial cells by a Rac1-dependent PI3K/Akt pathway. J Biol Chem. 2011;286:10483-94. 
37. Zhang F, Ma H, Wang ZL, Li WH, Liu H, Zhao YX. The PI3K/AKT/mTOR pathway regulates autophagy to induce apoptosis of alveolar epithelial cells in chronic obstructive pulmonary disease caused by PM2.5 particulate matter. J Int Med Res. 2020;48:300060520927919.

38. Chen S, Zhang Z, Chen L, Zhang J. miRNA-101-3p.1 as an independent diagnostic biomarker aggravates chronic obstructive pulmonary disease via activation of the EGFR/PI3K/AKT signaling pathway. Mol Med Rep. 2019;20:4293-302.

39. Birukova AA, Fu P, Xing J, Birukov KG. Rap1 mediates protective effects of iloprost against ventilatorinduced lung injury. Journal of applied physiology (Bethesda, Md: 1985). 2009; 107: 1900-10.

40. Bivona TG, Philips MR. Analysis of Ras and Rap activation in living cells using fluorescent Ras binding domains. Methods. 2005;37:138-45.

41. Kovacs-Kasa A, Kim KM, Cherian-Shaw M, Black SM, Fulton DJ, Verin AD. Extracellular adenosineinduced Rac1 activation in pulmonary endothelium: Molecular mechanisms and barrier-protective role. Journal of cellular physiology. 2018;233:5736-46.

42. Birukova AA, Zagranichnaya T, Fu P, Alekseeva E, Chen W, Jacobson JR, et al. Prostaglandins PGE(2) and PGI(2) promote endothelial barrier enhancement via PKA- and Epac1/Rap1-dependent Rac activation. Experimental cell research. 2007;313:2504-20.

43. Tan DBA, Ito J, Peters K, Livk A, Lipscombe RJ, Casey TM, et al. Protein Network Analysis Identifies Changes in the Level of Proteins Involved in Platelet Degranulation, Proteolysis and Cholesterol Metabolism Pathways in AECOPD Patients. Copd. 2020;17:29-33.

44. Jia J, Conlon TM, Sarker RS, Taşdemir D, Smirnova NF, Srivastava B, et al. Cholesterol metabolism promotes B-cell positioning during immune pathogenesis of chronic obstructive pulmonary disease. EMBO molecular medicine. 2018; 10.

45. Yao X, Gordon EM, Figueroa DM, Barochia AV, Levine SJ. Emerging Roles of Apolipoprotein E and Apolipoprotein A-l in the Pathogenesis and Treatment of Lung Disease. Am J Respir Cell Mol Biol. 2016;55:159-69.

46. Boué S, De León H, Schlage WK, Peck MJ, Weiler H, Berges A, et al. Cigarette smoke induces molecular responses in respiratory tissues of $\mathrm{ApoE}(-/-)$ mice that are progressively deactivated upon cessation. Toxicology. 2013;314:112-24.

47. Goldklang M, Golovatch P, Zelonina T, Trischler J, Rabinowitz D, Lemaître V, et al. Activation of the TLR4 signaling pathway and abnormal cholesterol efflux lead to emphysema in ApoE-deficient mice. American journal of physiology Lung cellular molecular physiology. 2012;302:L1200-8.

48. Laura D, Alessia DC, Gallo A, Bruckert E, Marcello A. ApoCIII: A multifaceted protein in cardiometabolic disease. Metabolism: clinical and experimental. 2020: 154395.

49. Cheng X, Yamauchi J, Lee S, Zhang T, Gong Z, Muzumdar R, et al. APOC3 Protein Is Not a Predisposing Factor for Fat-induced Nonalcoholic Fatty Liver Disease in Mice. J Biol Chem. 2017;292:3692-705.

50. Milonas D, Tziomalos K. Experimental therapies targeting apolipoprotein C-III for the treatment of hyperlipidemia - spotlight on volanesorsen. Expert opinion on investigational drugs. 2019; 28: 389- 
94.

51. Haegens A, Schols AM, Gorissen SH, van Essen AL, Snepvangers F, Gray DA, et al. NF-kB activation and polyubiquitin conjugation are required for pulmonary inflammation-induced diaphragm atrophy. American journal of physiology Lung cellular molecular physiology. 2012;302:L103-10.

52. Taivassalo T, Hussain SN. Contribution of the Mitochondria to Locomotor Muscle Dysfunction in Patients With COPD. Chest. 2016;149:1302-12.

53. Hussain SN, Sandri M. Role of autophagy in COPD skeletal muscle dysfunction. Journal of applied physiology (Bethesda, Md: 1985). 2013; 114: 1273-81.

54. Dikshit A, Zhang JY. UBE2N plays a pivotal role in maintaining melanoma malignancy. Oncotarget. 2018;9:37347-8.

55. Uddin MA, Barabutis N. P53 in the impaired lungs. DNA Repair. 2020;95:102952.

56. Mizuno S, Ishizaki T, Kadowaki M, Akai M, Shiozaki K, Iguchi M, et al. p53 Signaling Pathway Polymorphisms Associated With Emphysematous Changes in Patients With COPD. Chest. 2017;152:58-69.

57. Xue H, Li MX. MicroRNA-150 protects against cigarette smoke-induced lung inflammation and airway epithelial cell apoptosis through repressing p53: MicroRNA-150 in CS-induced lung inflammation. Hum Exp Toxicol. 2018;37:920-8.

58. Li X, Zheng M, Pu J, Zhou Y, Hong W, Fu X, et al. Identification of abnormally expressed IncRNAs induced by PM2.5 in human bronchial epithelial cells. Bioscience reports. 2018; 38.

59. Gu C, Li Y, Liu J, Ying X, Liu Y, Yan J, et al. LncRNA-mediated SIRT1/FoxO3a and SIRT1/p53 signaling pathways regulate type II alveolar epithelial cell senescence in patients with chronic obstructive pulmonary disease. Mol Med Rep. 2017;15:3129-34.

60. Hou Y, Wang Z, Huang S, Sun C, Zhao J, Shi J, et al. SKA3 Promotes tumor growth by regulating CDK2/P53 phosphorylation in hepatocellular carcinoma. Cell death disease. 2019;10:929.

\section{Figures}




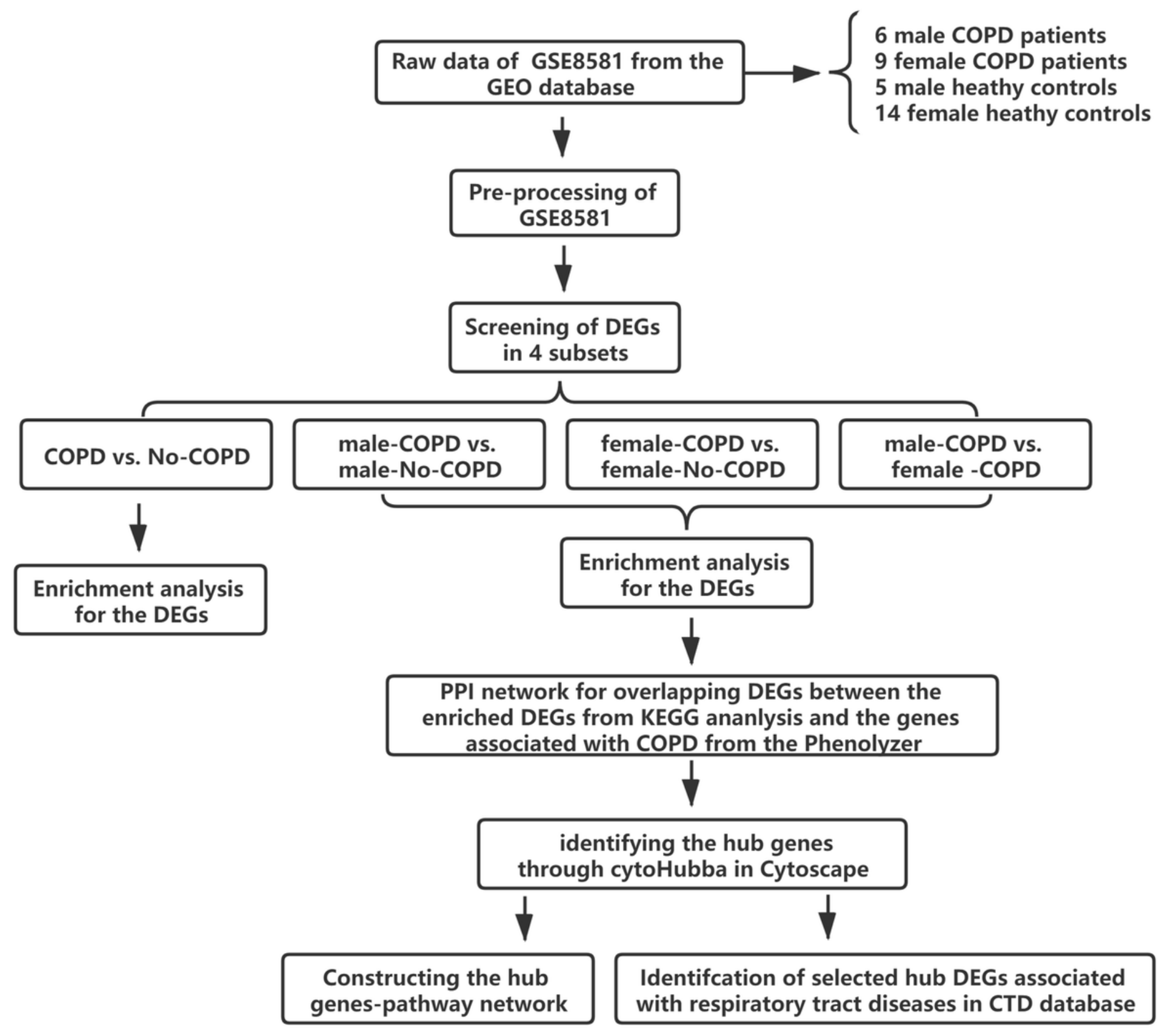

Figure 1

A flow chart of the analyses used in this study. 

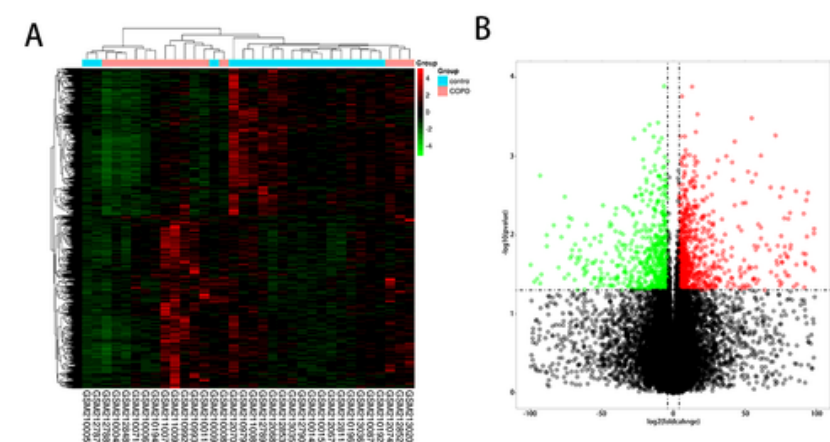

C $\quad$ D
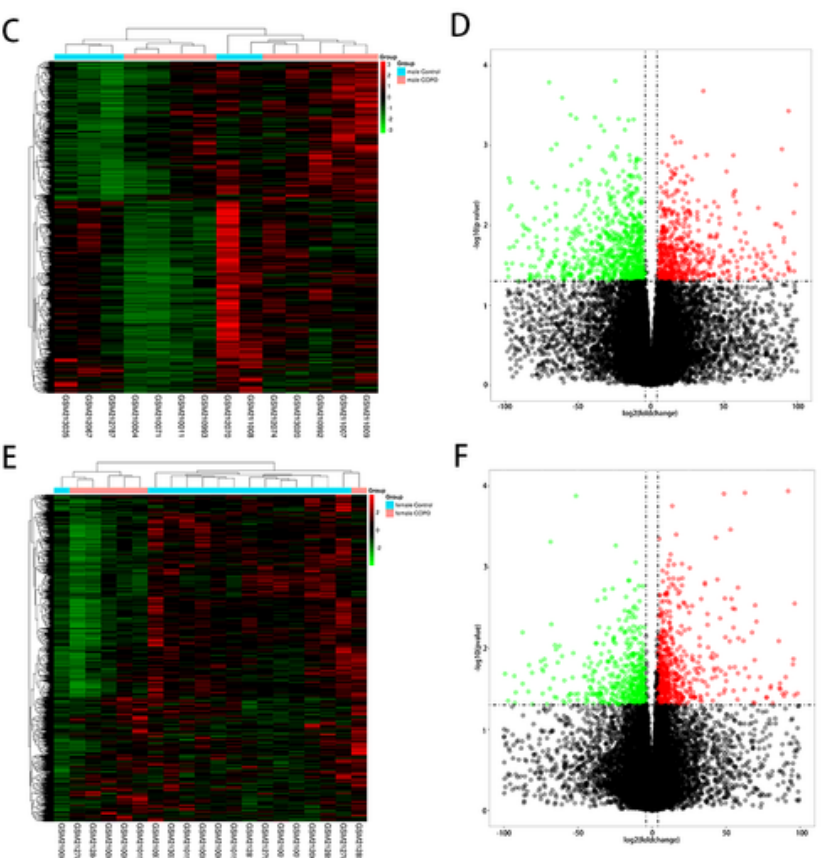

G

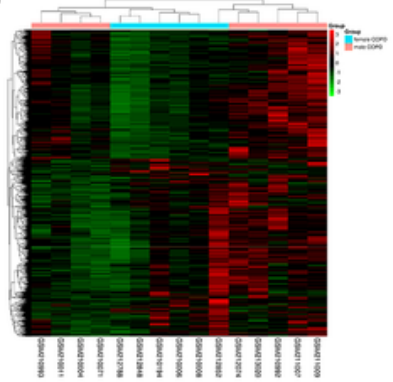

$\mathrm{H}$
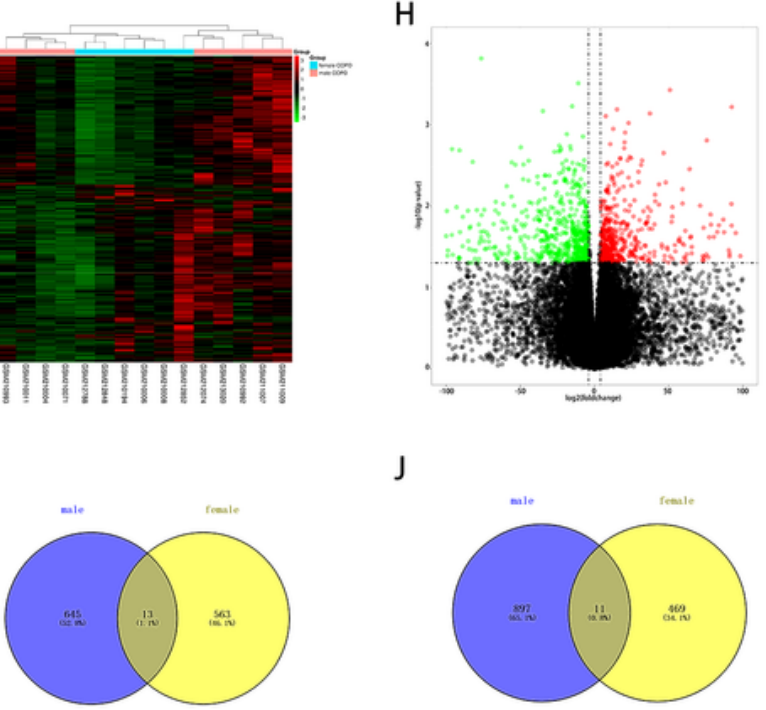

J

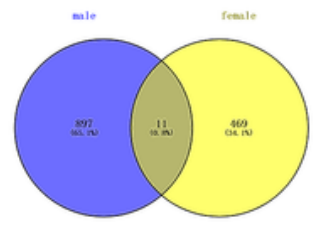

Figure 2

Data normalization. Box plots of gene expression in GSE8581 (A) before and (B) after normalization. 
A

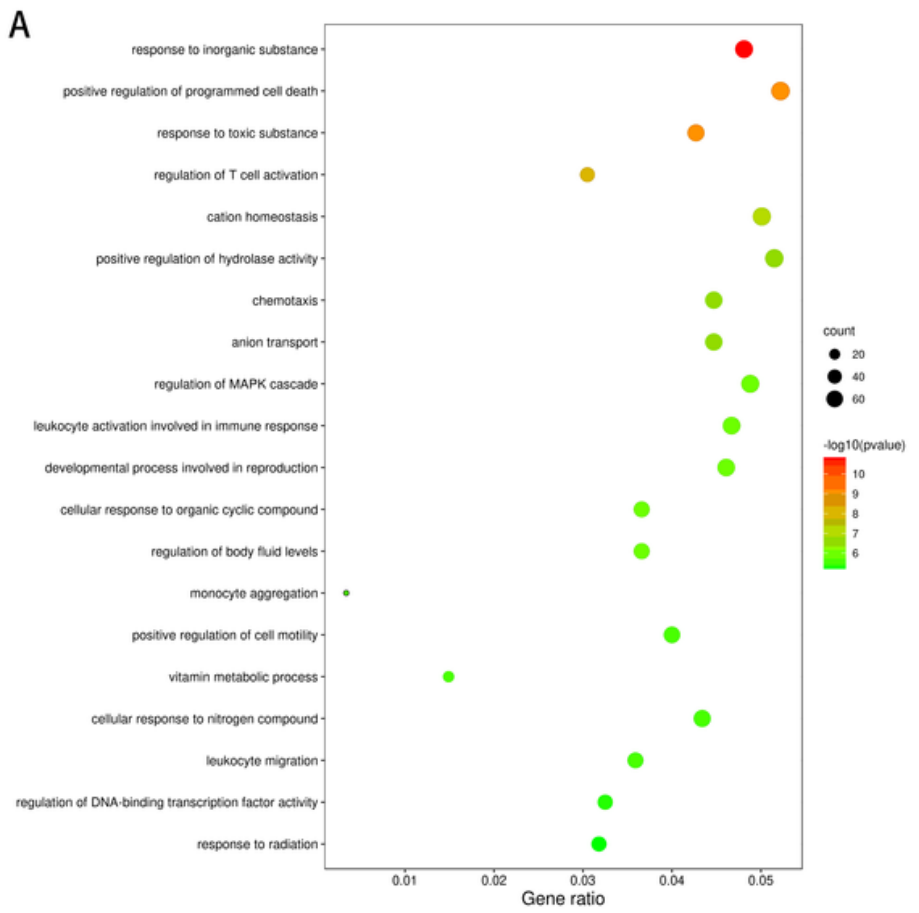

B

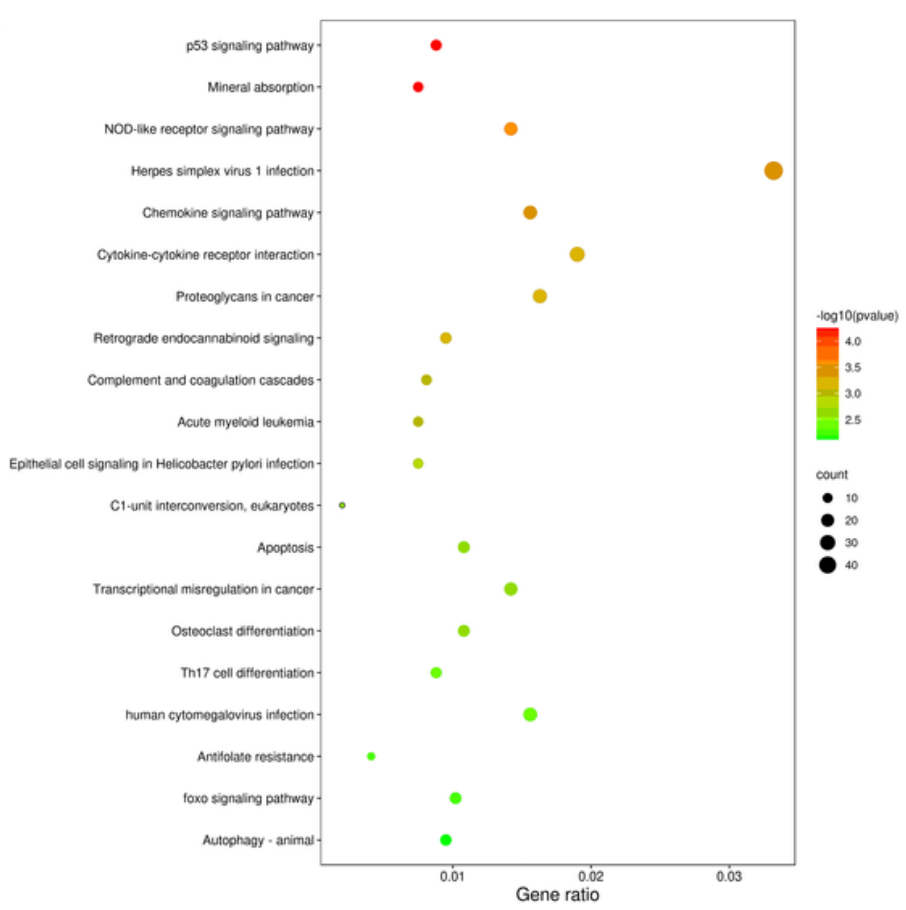

Figure 3

(A) The GO annotations for biological process and (B) KEGG pathway analysis of the 20 most significant GO enrichment terms for DEGs between COPD patients and healthy controls. The horizontal axis represents the ratio of enriched genes in the selected category to all genes analyzed in the GO or KEGG enrichment analyses while the vertical axis represents enriched GO or KEGG categories. 
A

B

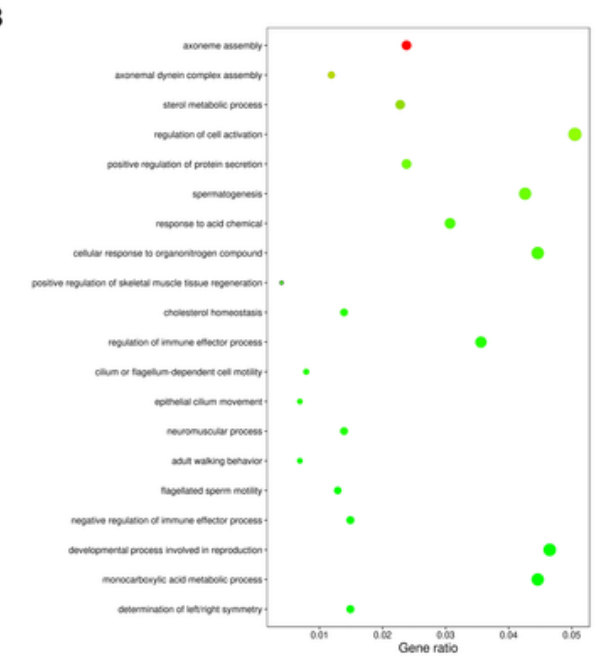

C
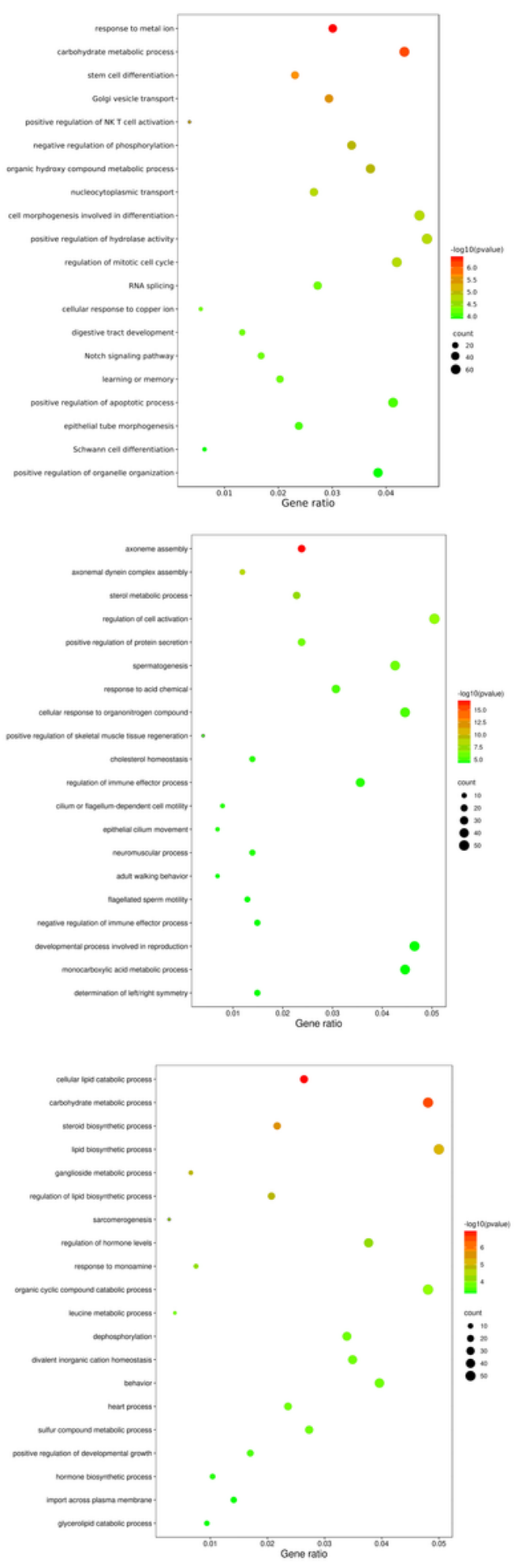

D

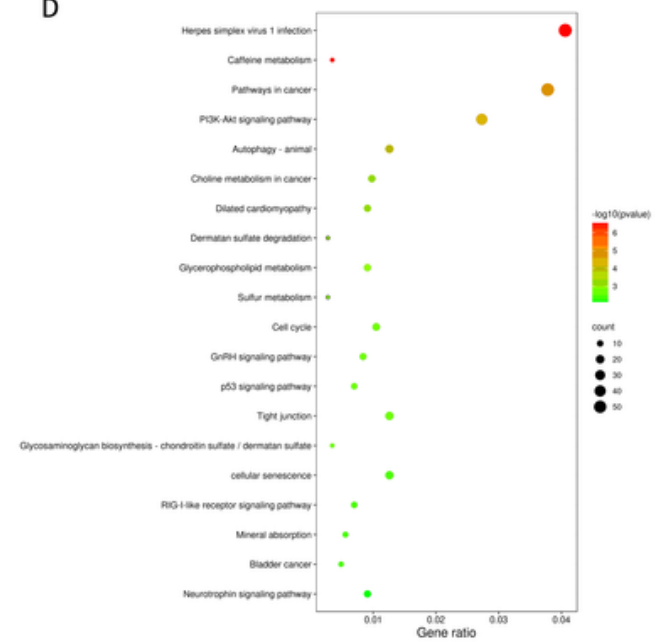

$E$

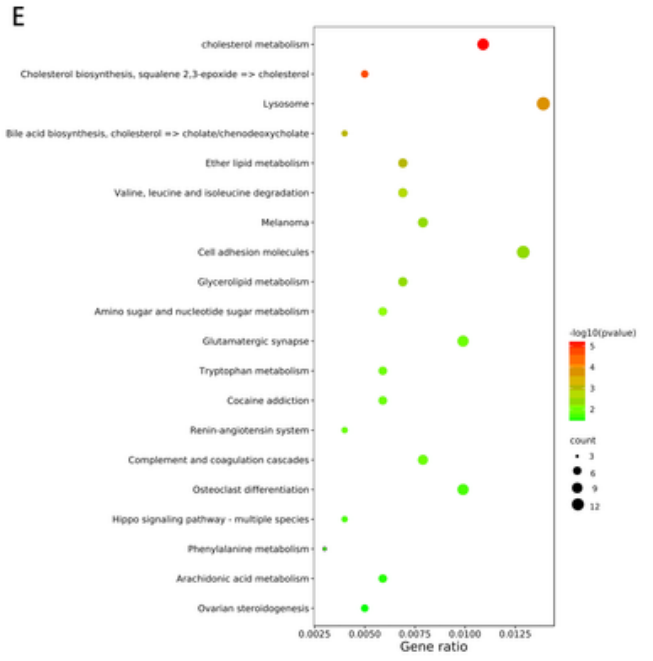

$\mathrm{F}$

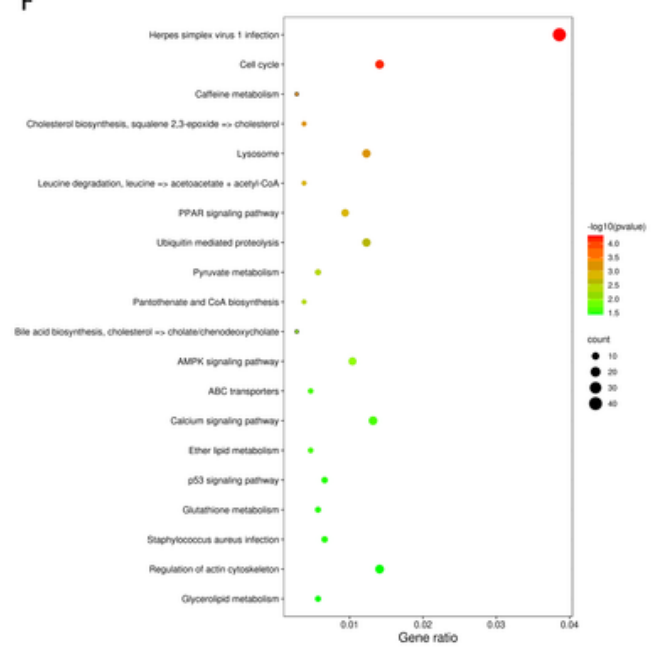

Figure 4

(A; B) Hierarchical clustering analysis and volcano plot of DEGs between COPD patients and healthy controls, (C; D)between healthy male controls and male COPD patients, $(E ; F)$ between healthy female controls and female COPD samples, $(G ; H)$ DEGs between the male COPD samples and female COPD samples. The relative levels of gene expression are represented using a color scale: red represents high levels while blue represents low levels. The green dots indicate down-regulated DEGs while the red dots 
represent up-regulated DEGs in the volcano plot. Venn diagrams of DEGs in female and male COPD patients, including (I) up-regulated DEGs and (J) down-regulated DEGs.

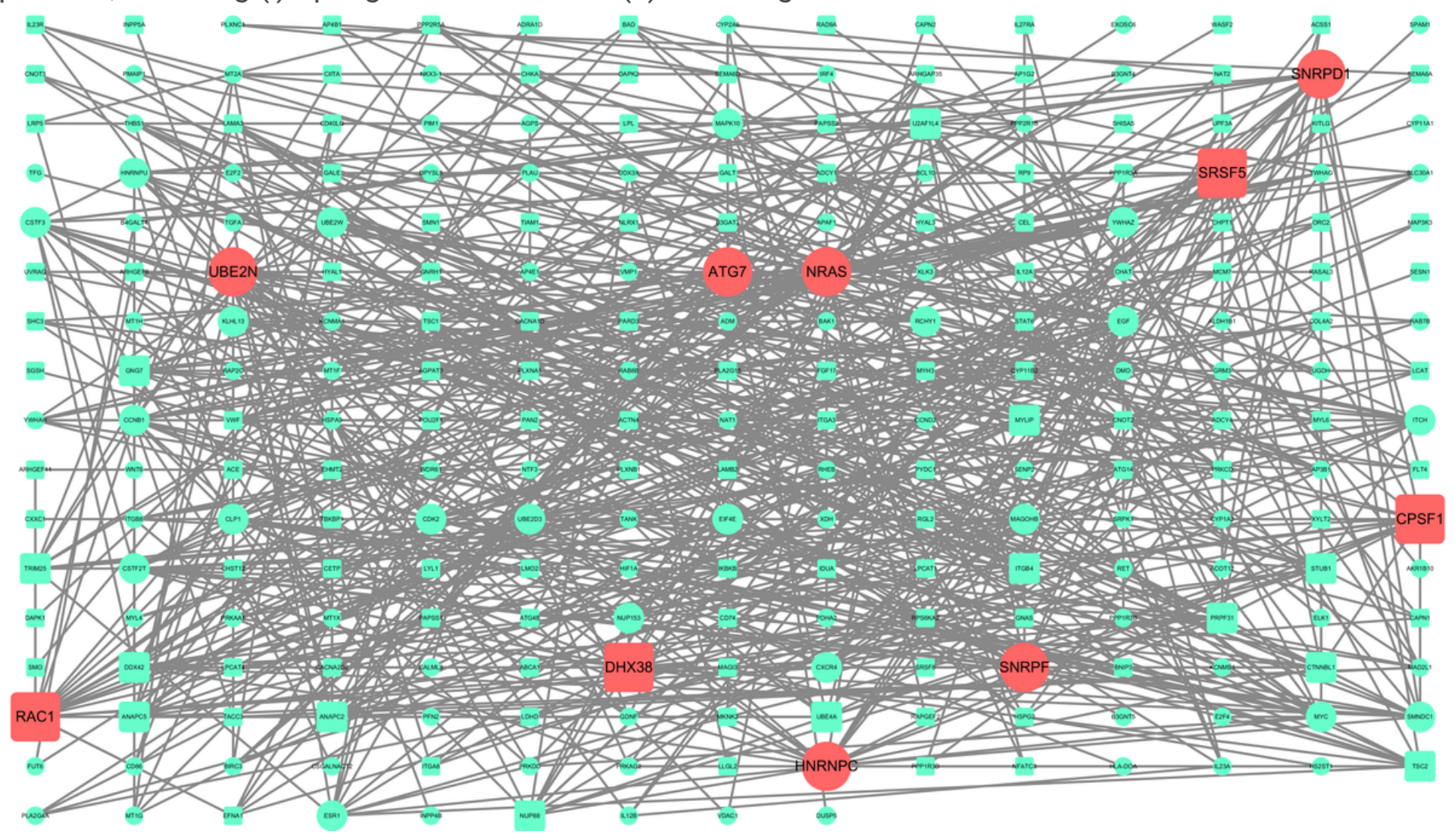

\section{Figure 5}

The GO annotations for biological process and KEGG pathway analysis of 20 most significant GO enrichment terms for DEGs $(A ; D)$ between healthy male controls and male COPD patients $(B ; E)$ between healthy female controls and female COPD samples, (C; F) DEGs between the male COPD samples and female COPD samples. The horizontal axis represents the ratio of enriched genes in the selected category to all genes analyzed in the GO or KEGG enrichment analyses while the vertical axis represents enriched GO or KEGG categories. 


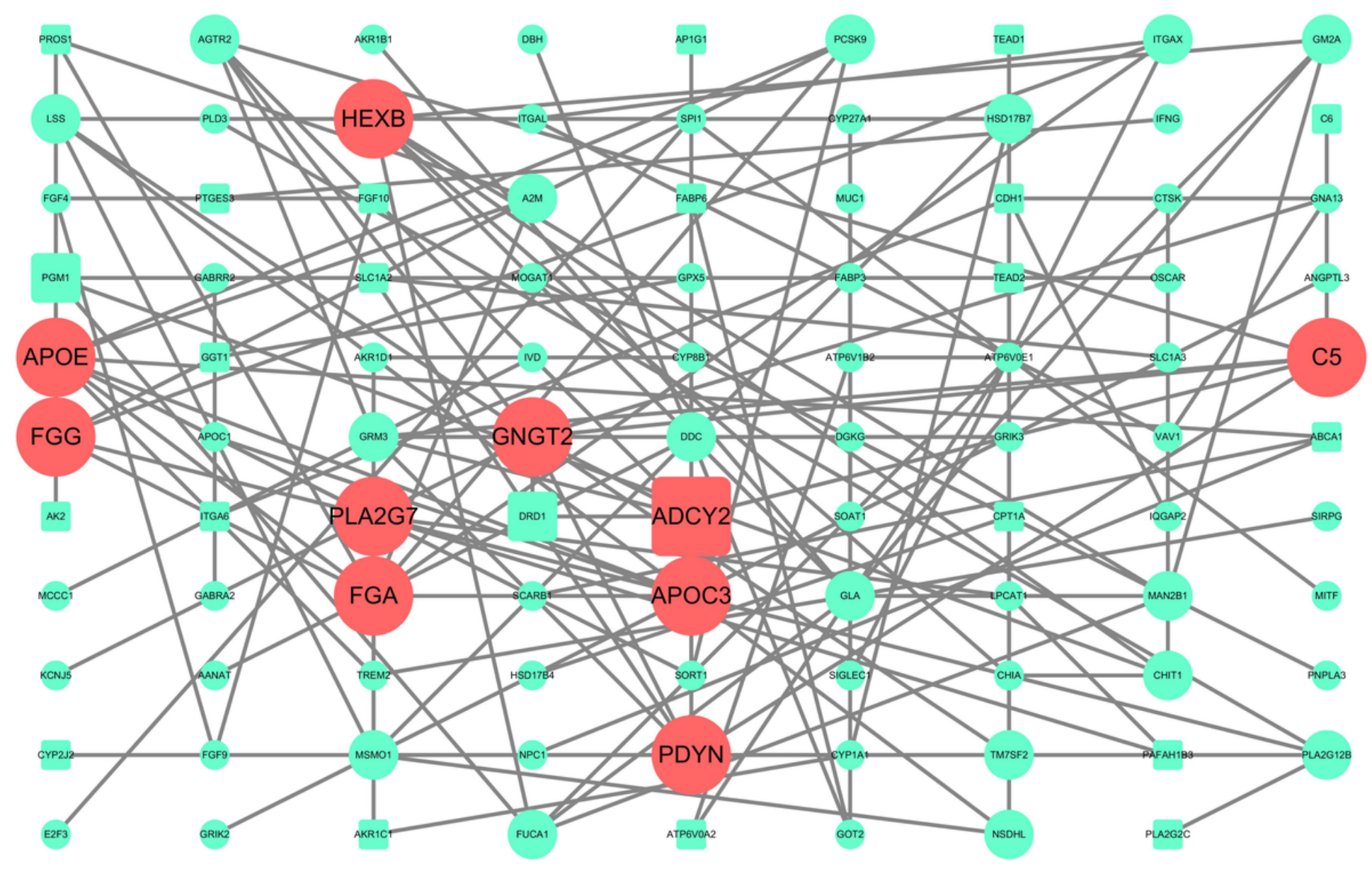

Figure 6

The Protein-protein interaction network of DEGs between healthy male controls and male COPD patients. The circular nodes represent upregulated genes and the square nodes represent downregulated genes. The higher the degree, the larger the shape. The red nodes had a higher degree and were identified as hub genes. 


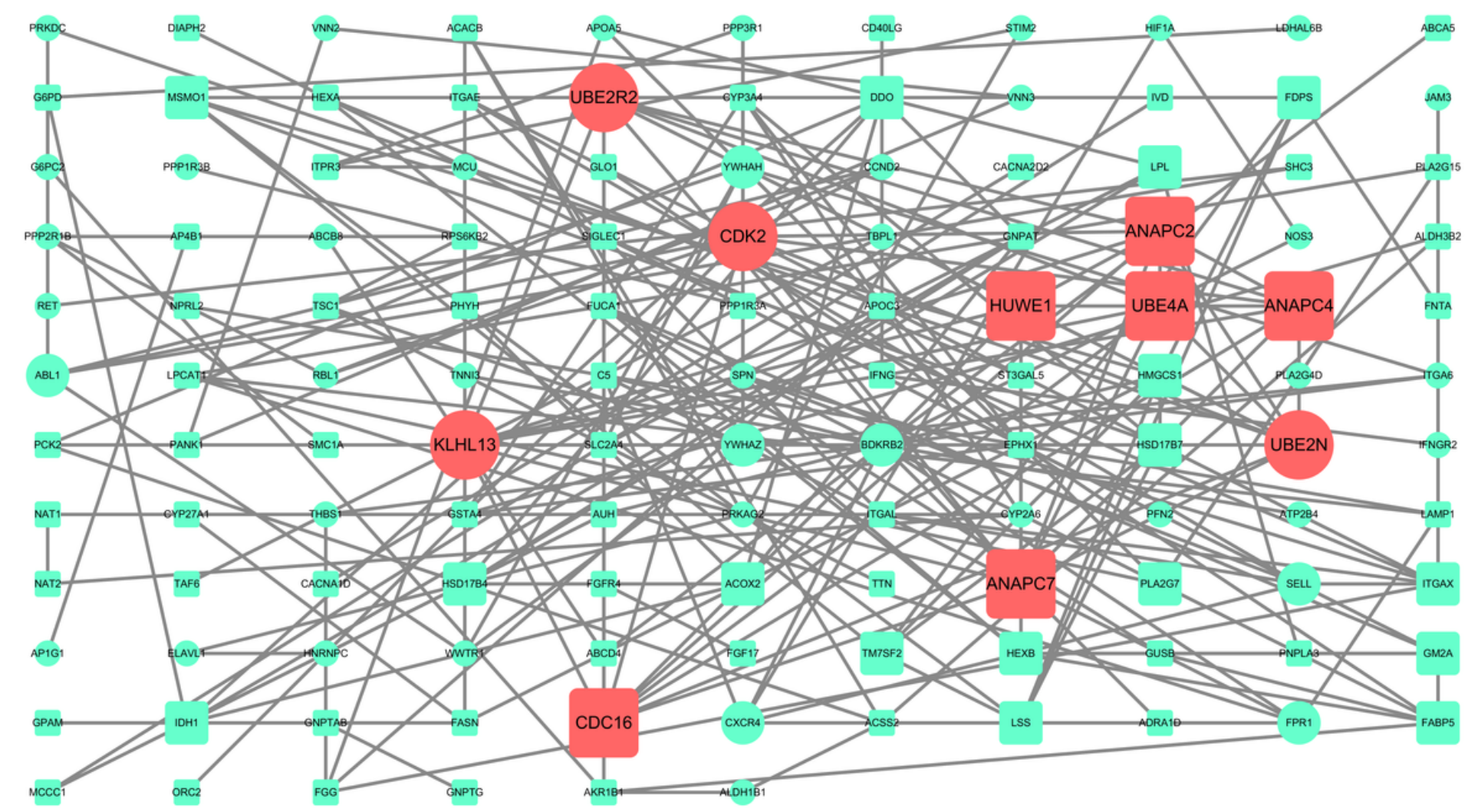

Figure 7

The Protein-protein interaction network of DEGs between healthy female controls and female COPD patients. The circular nodes represent upregulated genes and the square nodes represent downregulated genes. The higher the degree, the larger the shape. The red nodes had a higher degree and were identified as hub genes

A

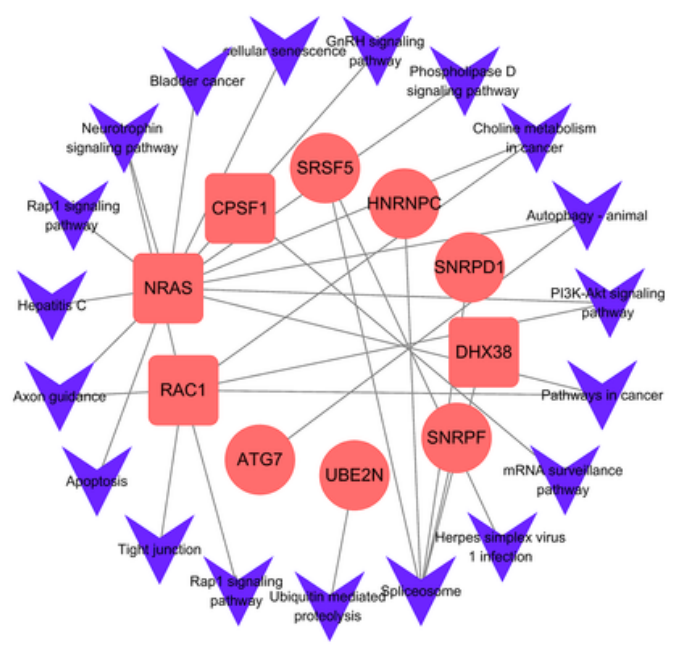

B

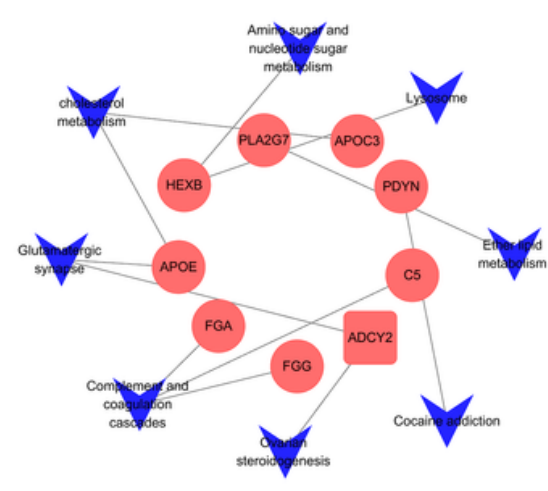

C

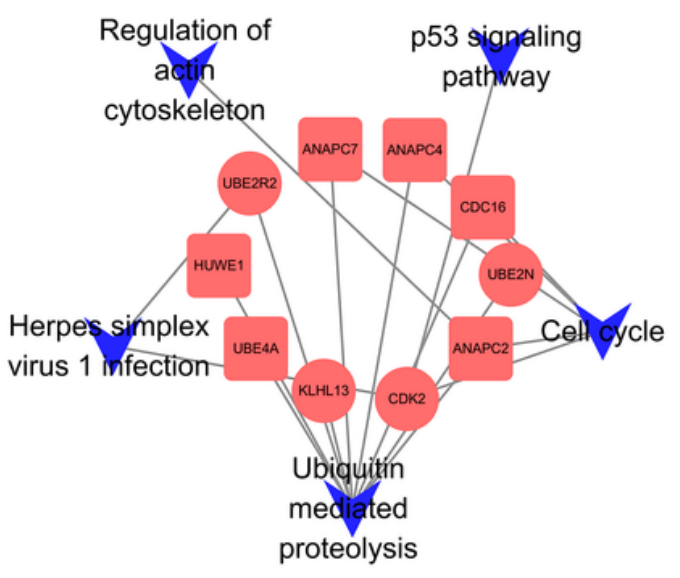

\section{Figure 8}


The Protein-protein interaction network of DEGs between male COPD patients and female COPD patients. The circular nodes represent upregulated genes and the square nodes represent downregulated genes. The higher the degree, the larger the shape. The red nodes had a higher degree and were identified as hub genes.

\section{Image not available with this version}

\section{Figure 9}

The hub genes-related pathway network for (A) male-COPD vs. male -No-COPD, (B) female-COPD vs. female-No-COPD and (C) male-COPD vs. female-COPD. The $V$ shape indicates the pathways while the red nodes denote the hub genes related to the pathways. The circular red nodes represent upregulated genes and the square red nodes represent downregulated genes.

\section{Image not available with this version}

\section{Figure 10}

The top 20 respiratory tract diseases related to (A) NRAS, (B) RAC1, (C) APOE, (D) APOC3, (E) UBE2N and (F) CDK2, based on the CTD database. * related to COPD.

\section{Supplementary Files}

This is a list of supplementary files associated with this preprint. Click to download.

- SupplementaryFigure1.tif

- SupplementaryFigure2.tif

- SupplementaryFigure3.tif

- SupplementaryTableS14.xlsx

- SupplementaryTableS15.xIsx

- SupplemnataryTableS1.xls

- SupplemnataryTableS11.xIsx

- SupplemnataryTableS13.xlsx 
- SupplemnataryTableS2.xls

- SupplemnataryTableS3.xls

- SupplemnataryTableS4.xls

- SupplemnataryTableS5.xlsx

- SupplemnataryTableS6.xlsx

- SupplemnataryTableS8.xlsx

- SupplemnataryTableS10.xlsx

- SupplemnataryTableS12.xIsx

- SupplemnataryTableS7.xlsx

- SupplemnataryTableS9.xIsx 